\title{
Inhibiting NLRP3 inflammasome activation prevents copper-induced neuropathology in a murine model of Wilson's disease
}

Jianjian Dong ${ }^{1,2,3}$, Xun Wang ${ }^{3,4}$, Chenchen Xu ${ }^{3,4}$, Manli Gao ${ }^{3,4}$, Shijing Wang ${ }^{3,4}$, Jin Zhang ${ }^{1}$, Haiyang Tong ${ }^{1}$, Lulu Wang ${ }^{1}$, Yongzhu Han ${ }^{3,4}$, Nan Cheng ${ }^{3,4}$ and Yongsheng $\operatorname{Han}^{3,4}$

\begin{abstract}
Wilson's disease (WD) is an inherited disorder characterized by excessive accumulation of copper in the body, particularly in the liver and brain. In the central nervous system (CNS), extracellular copper accumulation triggers pathological microglial activation and subsequent neurotoxicity. Growing evidence suggests that levels of inflammatory cytokines are elevated in the brain of murine WD models. However, the mechanisms associated with copper deposition to neuroinflammation have not been completely elucidated. In this study, we investigated how the activation of NLR family pyrin domain containing 3 (NLRP3) inflammasome contributes to copper-mediated neuroinflammation in an animal model of WD. Elevated levels of interleukin-1 $\beta$, interleukin-18, interleukin-6, and tumor necrosis factor-a were observed in the sera of WD patients and toxic milk (TX) mice. The protein levels of inflammasome adaptor molecule apoptosis-associated speck-like protein containing a C-terminal caspase recruitment domain (ASC), cleaved caspase-1, and interleukin-1 $\beta$ were upregulated in the brain regions of the TX mice. The NLRP3 inflammasome was activated in the TX mice brains. Furthermore, the activation of NLRP3 inflammasome was noted in primary microglia treated with $\mathrm{CuCl}_{2}$, accompanied by the increased levels of cleaved caspase-1, ASC, and interleukin1 $\beta$. Blocking NLRP3 inflammasome activation with siNlrp3 or MCC950 reduced interleukin-1 $\beta$ and interleukin-18 production, thereby effectively mitigating cognitive decline, locomotor behavior impairment, and neurodegeneration in TX mice. Overall, our study demonstrates the contribution of copper overload-mediated activation of NLRP3 inflammasome to progressive neuropathology in the CNS of a murine model of WD. Therefore, blockade of the NLRP3 inflammasome activation could be a potential therapeutic strategy for WD.
\end{abstract}

\section{Introduction}

Wilson's disease (WD) is an autosomal recessive disorder related to copper metabolism that is caused by mutations in $A T P 7 B$, which mediates the binding of copper to ceruloplasmin and the excretion of copper into bile $^{1}$. ATP7B dysfunction results in excess copper deposits in various organs, including the liver, brain, and eyes.

\footnotetext{
Correspondence: Nan Cheng (NanCheng111065@ahtcm.edu.cn) or Yongsheng Han (hyysp@ahtcm.edu.cn)

${ }^{1}$ High Magnetic Field Laboratory, Hefei Institutes of Physical Science, Chinese Academy of Sciences, Hefei 230031, P. R. China

2University of Science and Technology of China, Hefei 230026, P. R. China

Full list of author information is available at the end of the article

Edited by A. Verkhratsky
}

Growing evidence indicates that neuroinflammation is common in patients with $\mathrm{WD}^{2-4}$. The pathological changes in the central nervous system (CNS) associated with WD include astrogliosis, demyelination, and tissue disintegration ${ }^{5,6}$. However, the mechanism by which copper overload contributes to progressive neurodegeneration and neuroinflammation in WD remains elusive.

Inflammasomes are large multiprotein complexes that act as intracellular sensors for a variety of dangerassociated molecular patterns and pathogen-associated molecular patterns (PAMPs) $)^{7,8}$. Canonical inflammasomes are composed of an inflammasome sensor protein bound to pro-caspase-1, usually via an adaptor molecule

\section{(c) The Author(s) 2021}

(c) (i) Open Access This article is licensed under a Creative Commons Attribution 4.0 International License, which permits use, sharing, adaptation, distribution and reproduction cc) in any medium or format, as long as you give appropriate credit to the original author(s) and the source, provide a link to the Creative Commons license, and indicate if changes were made. The images or other third party material in this article are included in the article's Creative Commons license, unless indicated otherwise in a credit line to the material. If material is not included in the article's Creative Commons license and your intended use is not permitted by statutory regulation or exceeds the permitted use, you will need to obtain permission directly from the copyright holder. To view a copy of this license, visit http://creativecommons.org/licenses/by/4.0/. 
apoptosis-associated speck-like protein containing a Cterminal caspase recruitment domain (ASC). Inflammasome sensor proteins include nucleotide binding oligomerization domain (NOD)-like receptor family, pyrin domain-containing protein (NLRP) 1, NLRP3, NLRP6, neuronal apoptosis inhibitory protein (NAIP)/ NLR containing C-terminal caspase recruitment domain protein 4 (NLRC4), absent in melanoma 2 (AIM2), and pyrin; each of these responds to specific pathogen- and dangerassociated signals indicative of infection or cellular damage, triggering sensor protein oligomerization and the recruitment of $\mathrm{ASC}^{9,10}$. Activation of the canonical inflammasomes promotes caspase-1-dependent maturation of interleukin (IL)- $1 \beta$ and IL-18 ${ }^{11,12}$.

IL-1 $\beta$, a proinflammatory cytokine that is upregulated in many CNS inflammatory conditions, including Alzheimer's disease, Parkinson's disease, multiple sclerosis, and other neurodegenerative disorders, is a master regulator of immune responses in the $\mathrm{CNS}^{13,14}$. It is capable of activating an innate immune response by inducing the expression of various inflammatory cytokines and chemokines and eliciting leukocyte recruitment ${ }^{15,16}$. As a key driver of the neuroinflammatory process, IL-1 $\beta$ is expressed by many different cell types, including microglia, astrocytes, endothelial cells, recruited leukocytes, and oligodendrocytes, within the CNS. The IL- $1 \beta$ precursor (namely, pro-IL-1 $\beta$ ) is not constitutively expressed and is induced by NF- $\mathrm{KB}$-mediated transcription activation. In addition, pro-IL-1 $\beta$, is not biologically active; however, it requires proteolytic processing by caspase- 1 , resulting in the secretion of mature IL- $1 \beta^{17,18}$. Caspase- 1 , the predominant IL-1 processing protease, is abundantly present in the microglia as a proenzyme and is typically activated by inflammasomes ${ }^{19-21}$.

Although copper plays an essential role in the development of healthy nerves and other physiological functions in the $\mathrm{CNS}^{22}$, the accumulation of excessive $\mathrm{Cu}^{2+}$ amounts can lead to neuroinflammatory injury ${ }^{23,24}$. WD results from copper accumulation in the liver and brain caused by mutations in $A T P 7 B$, encoding a copper transporter. Copper accumulation in rodent brains induces WD features and the recruitment of inflammatory cells into the affected brain region ${ }^{4}$. Copper is specifically required for inflammasome activation in macrophages, as the depletion of bioavailable copper results in an attenuated caspase-1-dependent inflammation and reduced susceptibility to lipopolysaccharide (LPS)-induced septic $\operatorname{shock}^{25}$. Therefore, we hypothesized that copper accumulation activates NLRP3 inflammasome to mediate neuroinflammation in the brain.

The present study aimed to investigate the role of inflammasome activation in the initiation of WD using toxic milk (TX) mice, an animal model for WD. Herein, we show that the activation of NLRP3 inflammasome in the brain of TX mice caused copper-induced neuropathology. siRNA silencing or pharmacologic blockade of NLRP3 inflammasome activation inhibited the microglial activation and release of proinflammatory cytokines in the CNS, thereby improving cognitive function in TX mice. Altogether, our study highlighted the importance of NLRP3 activation as a pathological mechanism involved in the development of WD and suggested that inhibiting of the activation of NLRP3 inflammasome might be an ideal therapeutic strategy to treat CNS complications in WD.

\section{Results}

Activation of the NLRP3 inflammasome is associated with WD

To determine if inflammation and inflammasome activation are involved in the development of WD, we first compared the levels of inflammatory cytokines in the sera of WD patients with those in healthy controls. Our data revealed significantly higher levels of circulating IL-1 $\beta$, IL-18, IL- 6 , and TNF- $\alpha$ in the sera of WD patients (Fig. 1a). In addition, elevated levels of IL-1 $\beta$, IL-18, IL-6, and TNF- $\alpha$ were also noted in the sera (Fig. 1b) and brain (Fig. 1c) of TX mice, as compared to those in WT mice. Furthermore, the levels of caspase-1, ASC, and IL-1 $\beta$ were remarkably elevated in the corpus striatum (Fig. 1d), hippocampus (Fig. S1a), cortex (Fig. S1b), and cerebellum (Fig. S1c) of the TX mice. Notably, the levels of NLRP3 were also remarkably higher in the corpus striatum (Fig. 1e) and hippocampus (Fig. S2a), while levels of NLRP2 and NLRP3 were elevated in the cortex (Fig. S2b) and cerebellum (Fig. S2c) of TX mice, compared to the WT mice. Mechanistically, the expression of Nlrp3, Casp1, and ASC was significantly elevated in the brains of TX mice, as compared with those in WT mice (Fig. S3). These findings demonstrated that inflammasome components are upregulated and subsequently activated in the early stages of neurodegeneration in TX mice.

\section{Copper induces NLRP3 activation and extracellular ASC release in microglia}

The activation of microglia plays a critical role in the development of chronic neuroinflammation. We examined whether copper triggered NLRP3 inflammasome activation in microglia. When primary microglia were treated with $\mathrm{CuCl}_{2}$, levels of IL-1 $\beta$ were elevated in the supernatant starting $12 \mathrm{~h}$ after treatment (Fig. 2a). Western blotting revealed elevated levels of NLRP3, cleaved caspase- 1 , ASC, and IL- $1 \beta$ in $\mathrm{CuCl}_{2}$-treated microglia in a time-dependent manner (Fig. 2b). To determine whether LPS is essential for copper-induced inflammasome activation, we examined whether $\mathrm{CuCl}_{2}$ alone could trigger IL- $1 \beta$ production in the absence of 

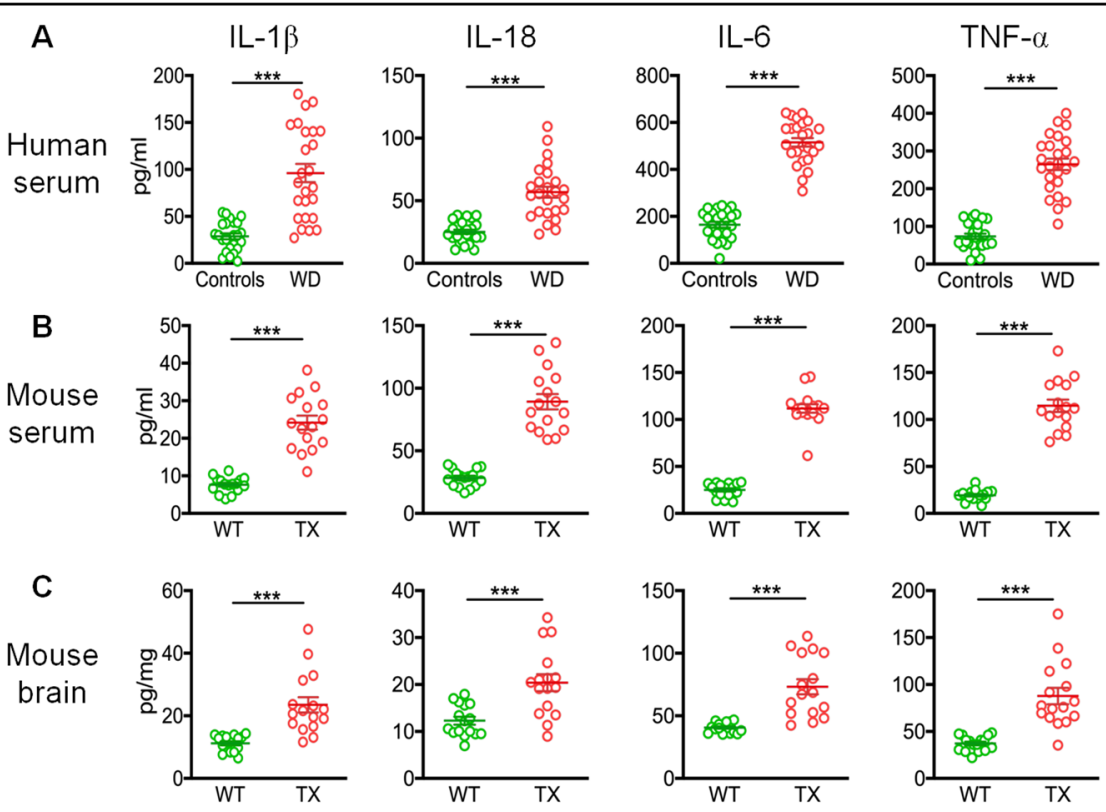

D
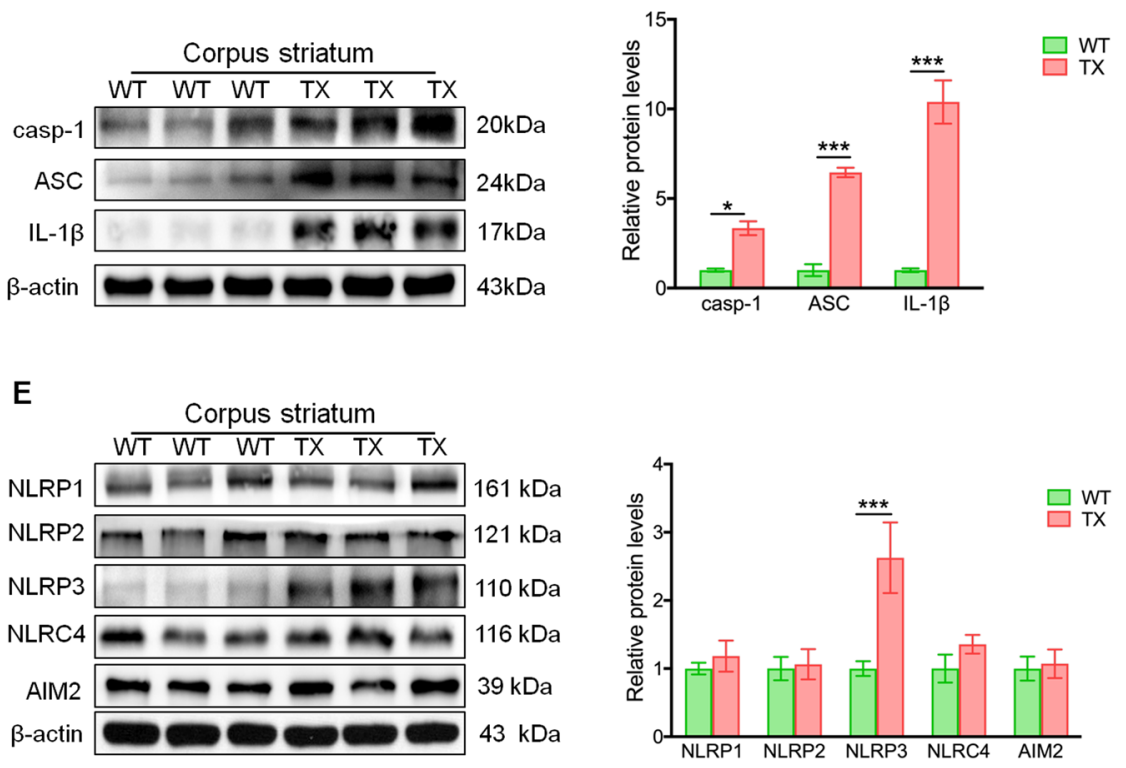

Fig. 1 Activation of NLRP3 inflammasome in patients with Wilson's disease (WD) and the WD animal model. a Levels of circulating interleukin (IL)-1 $\beta, I L-18, I L-6$, and tumor necrosis factor (TNF)- $a$ in the serum of healthy controls and patients with WD $(n=25)$. b Serum IL-1 $1 \beta, I L-18, I L-6$, and TNF-a levels in toxic milk (TX) mice and wild type (WT) mice $(\mathrm{pg} / \mathrm{ml})(n=16)$. $\mathbf{c} \| \mathrm{L}-1 \beta, \mathrm{IL}-18, \mathrm{LL}-6$, and TNF-a levels in the brain of TX mice and WT controls mice $(\mathrm{pg} / \mathrm{mg})(n=16)$. $\mathbf{d}$ Levels of cleaved caspase-1 (casp-1), ASC, and IL-1 $\beta$ in the corpus striatum of TX mice and WT mice were quantified using western blotting $(n=6)$. e Levels of NLRP1, NLRP2, NLRP3, NLRC4, and AIM2 levels in the corpus striatum of TX mice and WT controls were measured using western blotting $(n=6)$. Data are presented as mean \pm SEM; Student's $t$ test, ${ }^{*} P<0.05,{ }^{* * *} P<0.001$ versus the corresponding control or WT mice.

LPS. Surprisingly, $\mathrm{CuCl}_{2}$ alone was able to trigger the secretion of mature IL-1 $\beta$ (Fig. 2c) from the microglia in a time-dependent manner (Fig. 2d). Furthermore, increased production of mature IL-18, an essential inflammatory mediator, was also observed in $\mathrm{CuCl}_{2}$ treated microglia (Fig. 2e).
In vivo NLRP3 silencing suppresses the copper-induced inflammatory response

To determine the importance of the NLRP3 inflammasome in copper-dependent inflammation in vivo, the expression of Nlrp3 was interrupted by injecting lentivirus-enveloped siNlrp3 into TX mice through the 

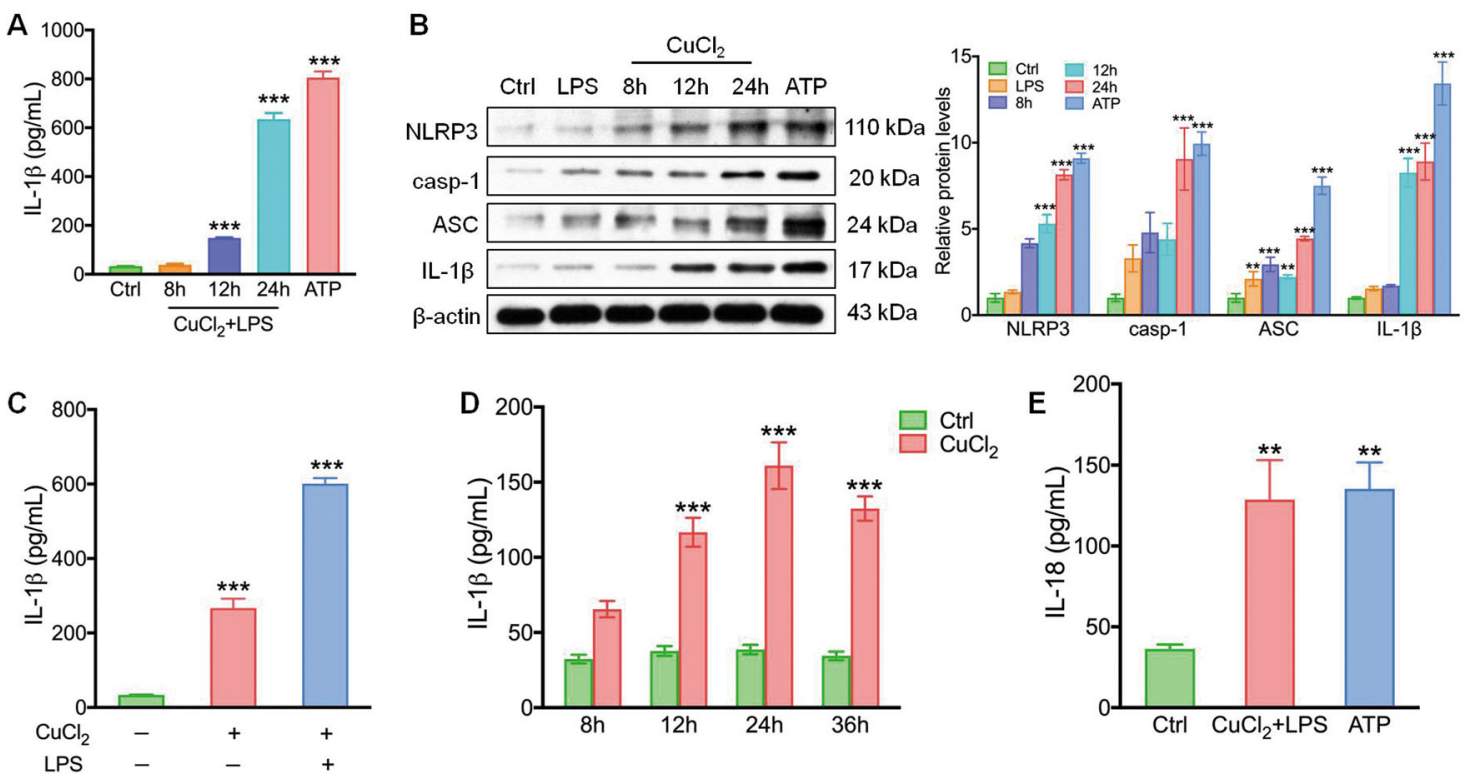

Fig. $2 \mathrm{CuCl}_{2}$ induces interleukin (IL-1 $\beta$ ) and IL-18 production and NLRP3 inflammasome activation. a Primary microglia were treated with $\mathrm{CuCl}_{2}(10 \mu \mathrm{M})$ and lipopolysaccharide (LPS, $\left.200 \mathrm{ng} / \mathrm{ml}\right)$, supernatants were collected at 8, 12, and $24 \mathrm{~h}$, and levels of IL-1 $\beta$ were quantified with ELISA; ATP $(5 \mathrm{mM})$ treatment served as the positive control. b Primary microglia were treated with $\mathrm{CuCl}_{2}$ for 8,12 , and $24 \mathrm{~h}$, and the protein levels of NLRP3, cleaved caspase-1 (casp-1), ASC, and IL-1 $\beta$ were quantified using western blotting; ATP (5 mM) treatment served as positive control. c Primary microglia were treated with or without $\mathrm{CuCl}_{2}(10 \mu \mathrm{M})$ and LPS $(200 \mathrm{ng} / \mathrm{ml})$ for $24 \mathrm{~h}$, and levels of $\mathrm{IL}-1 \beta$ were subsequently quantified using ELISA. d Primary microglia were treated with or without $\mathrm{CuCl}_{2}(10 \mu \mathrm{M})$ for $8,12,24$, and $36 \mathrm{~h}$, and the levels of IL-1 $\beta$ were quantified using ELISA. e The levels of IL-18 in microglia treated with $\mathrm{CuCl}_{2}$ and LPS for $24 \mathrm{~h}$. Data are presented as mean $\pm \mathrm{SEM}$; one-way ANOVA, two-way ANOVA, ${ }^{* *} P<0.01,{ }^{* * *} P<$ 0.001 versus the corresponding control group.

tail vein (Fig. 3a). As expected, reduced levels of the NLRP3 protein were noted in the corpus striatum (Fig. 3b) of mice treated with lentivirus-enveloped siNlrp3, as well as in other areas of the brain (Fig. S4). Furthermore, reduced protein levels of cleaved caspase-1, ASC, and IL$1 \beta$ were observed in the corpus striatum (Fig. 3c), hippocampus, cortex, and cerebellum (Fig. S4). Although the number of microglia in corpus striatum (Figs. 4a, b), hippocampus, cortex, and cerebellum (Fig. S5, S6) of siNlrp3-TX mice showed no significant differences with that of NC-treated TX mice, the levels of IL- $1 \beta$ and IL-18 (Fig. 4c, d) in TX-treated mice were significantly reduced following NLRP3 silencing. However, the levels of IL-6 and TNF- $\alpha$ showed no significant differences between siNlrp3-TX and NC-TX groups (Fig. 4c, d). Additionally, we examined whether siNlrp3 injection had beneficial effects on motor, emotional, and cognitive functions in TX mice. Interestingly, it increased the total distance and climbing behaviors (Fig. 4e), and improved motor function (Fig. 4f) and memory (Fig. 4g) of TX mice compared with NC-TX mice. However, siNlrp3 did not alter anxiety-like behavior in TX mice (Fig. 4h). Therefore, NLRP3 silencing suppresses copper-induced neuroinflammation and protects TX mice against progressive motor deficits and cognitive decline by eliminating NLRP3 inflammasome activation.

\section{MCC950 suppresses NLRP3 inflammasome activation in vitro}

To examine whether pharmacologic blockade of NLRP3 inflammasome activation could inhibit microglial activation, we examined the inhibitory effect of MCC950, a potent inhibitor of NLRP3, on the activation of primary microglia induced by $\mathrm{CuCl}_{2}$ in vitro (Fig. $5 \mathrm{a}$ ). $\mathrm{CuCl}_{2}$ stimuli increased the release of IL-1 $\beta$ and IL-18 in primary microglia. When added to the culture, MCC950 significantly reduced the levels of IL-1 $\beta$ and IL18 secreted by $\mathrm{CuCl}_{2}$-activated microglia (Fig. $5 \mathrm{~b}$ ). The protein levels of NLRP3, caspase-1, ASC, and IL- $1 \beta$ in $\mathrm{CuCl}_{2}$-induced microglia were also attenuated by MCC950 treatment (Fig. 5c). Furthermore, conditioned medium was collected from primary microglia after LPS priming and $\mathrm{CuCl}_{2}$ or MCC950 treatment and subsequently added to primary neuron cultures. Compared to the control group, the TUNEL-positive cell number was significantly elevated in the culture treated with $\mathrm{CuCl}_{2}$ conditional medium. MCC950 conditional medium treatment decreased TUNEL-positive cell numbers in the neuron culture (Fig. 5d). In addition, MCC950 conditional medium efficiently reduced the number of dead neuron induced by $\mathrm{CuCl}_{2}$ (Fig. 5e). Together, $\mathrm{MCC} 950$ is sufficient to block the $\mathrm{CuCl}_{2}$-mediated pathological activation of microglial NLRP3 inflammasome. 
A

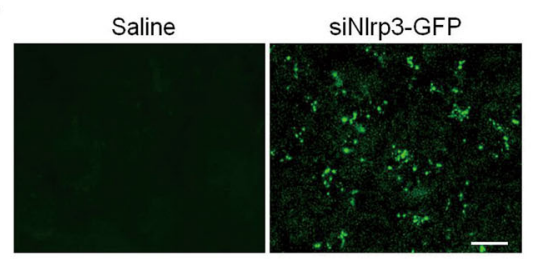

C

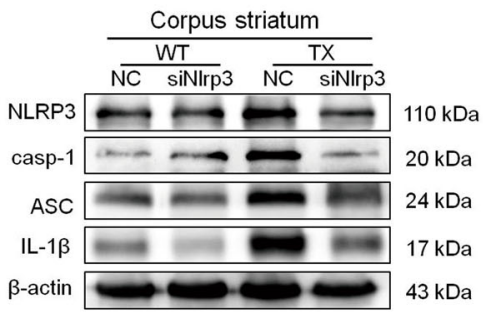

B
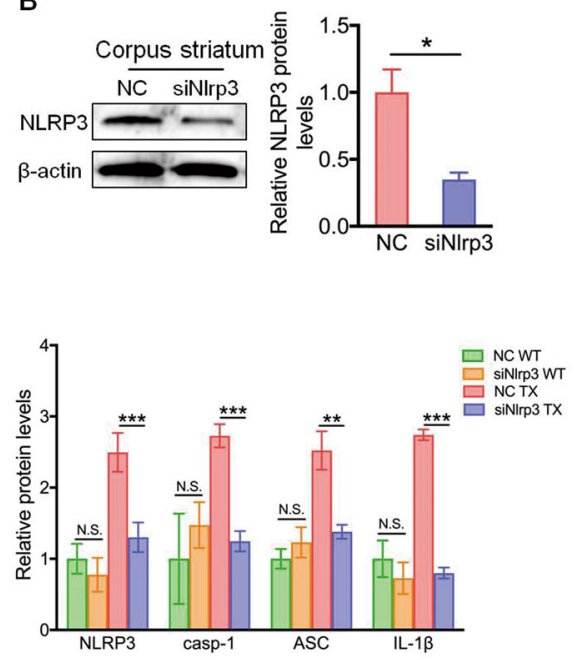

Fig. 3 Silencing NIrp3 expression reduced the caspase-1 and interleukin (IL)-1 $\beta$ protein levels in the Wilson's disease (WD) animal model. a Immunofluorescence staining of green fluorescent protein (GFP) in the corpus striatum of toxic milk (TX) mice injected with saline or Nlrp3 siRNA (siNlrp3). Scale bars, $50 \mu \mathrm{m}$. b Levels of NLRP3 in the brain of negative control siRNA (NC)- or siNlrp3-injected TX mice were measured using western blotting $(n=3)$. c Levels of NLRP3, cleaved caspase-1 (casp-1), ASC, and IL-1 $\beta$ in the corpus striatum of the NC- or siNlrp3-injected TX mice and WT mice were quantified using western blotting $(n=3)$. Data are presented as mean \pm SEM; Student's $t$ test, two-way ANOVA, ${ }^{*} P<0.05,{ }^{* *} P<0.01,{ }^{* * *} P<$ 0.001 versus the corresponding WT or NC group.

\section{MCC950 protects against neurodegeneration in TX mice}

Previous studies have demonstrated that orally administered MCC950 crosses the blood-brain barrier and affects biological functions in the brain ${ }^{26}$. Therefore, the effects of MCC950 treatment on cognitive function, locomotor behavior, and anxiety in TX mice were examined (Fig. 6a). MCC950 treatment significantly enhanced the total distance spontaneously traveled by TX mice, compared saline-treated TX mice (Fig. 6b). Additionally, the decreased instances of climbing behaviors and motor function in TX mice were reversed by MCC950 (Figs. 6b, c). Time spent in the center zone did not differ among various groups (Fig. 6d). Barnes maze experiments revealed that MCC950-treated TX mice exhibited improved memory and spatial learning, compared with the saline-treated TX mice (Fig. 6e).

Furthermore, the neuroprotective effects of MCC950 were accessed by Fluoro-Jade B staining and NeuN staining. Copper deposition induced a neuronal loss in the corpus striatum (Fig. 6f, g), as well as in the hippocampus, cortex, and cerebellum of TX mice (Figs. S7, S8). However, neuron loss in TX mice was alleviated by MCC 950 treatment (Figs. 6f, g). Therefore, MCC950 might protect against cognitive decline and locomotor behavior impairment by protecting neurodegeneration induced by copper deposition in TX mice.

\section{MCC950 reduces the activation of NLRP3 inflammasome in TX mice}

Next, we evaluated whether MCC950 had any impact on inflammasome activation. Mice treated with
MCC950 showed markedly decreased levels of NLRP3, cleaved caspase-1, and IL- $1 \beta$ in the corpus striatum (Fig. 7a) and other areas, including the hippocampus, cortex, and cerebellum (Fig. S9), as compared to those in salinetreated animals. MCC950 decreased the formation and release of ASC in TX mice compared to that in salinetreated animals (Fig. 7a and Fig. S9). The numbers of activated microglia were significantly increased in the brains of TX mice, whereas MCC950 treatment did not affect the number of activated microglia in different brain regions of TX mice (Fig. $7 \mathrm{~b}, \mathrm{c}$, and Figs. S10, S11). In addition, the levels of IL-1 $\beta$ and IL-18 in the serum and brain were reduced in MCC950-treated TX mice (Fig. 7d, e). However, the MCC950 administration did not affect the levels of IL- 6 and TNF- $\alpha$ in the serum and brain of TX mice (Fig. 7d, e). Altogether, these observations indicated that MCC950 suppresses neuroinflammation and protects against neurodegeneration in vivo by inhibiting NLRP3 inflammasome activation in TX mice (Fig. 8).

\section{Discussion}

WD is caused by abnormal copper metabolism in the liver due to a mutation in the gene encoding the copper transporting protein ATP7B. Current treatments for WD, including copper chelating agents, are designed to remove excess copper from tissues and organs ${ }^{27}$. Although these treatments have been used for decades, they are not entirely effective in many patients ${ }^{28}$. Therefore, the development of auxiliary therapies is required to improve the efficacy of copper chelation. Furthermore, chronic 


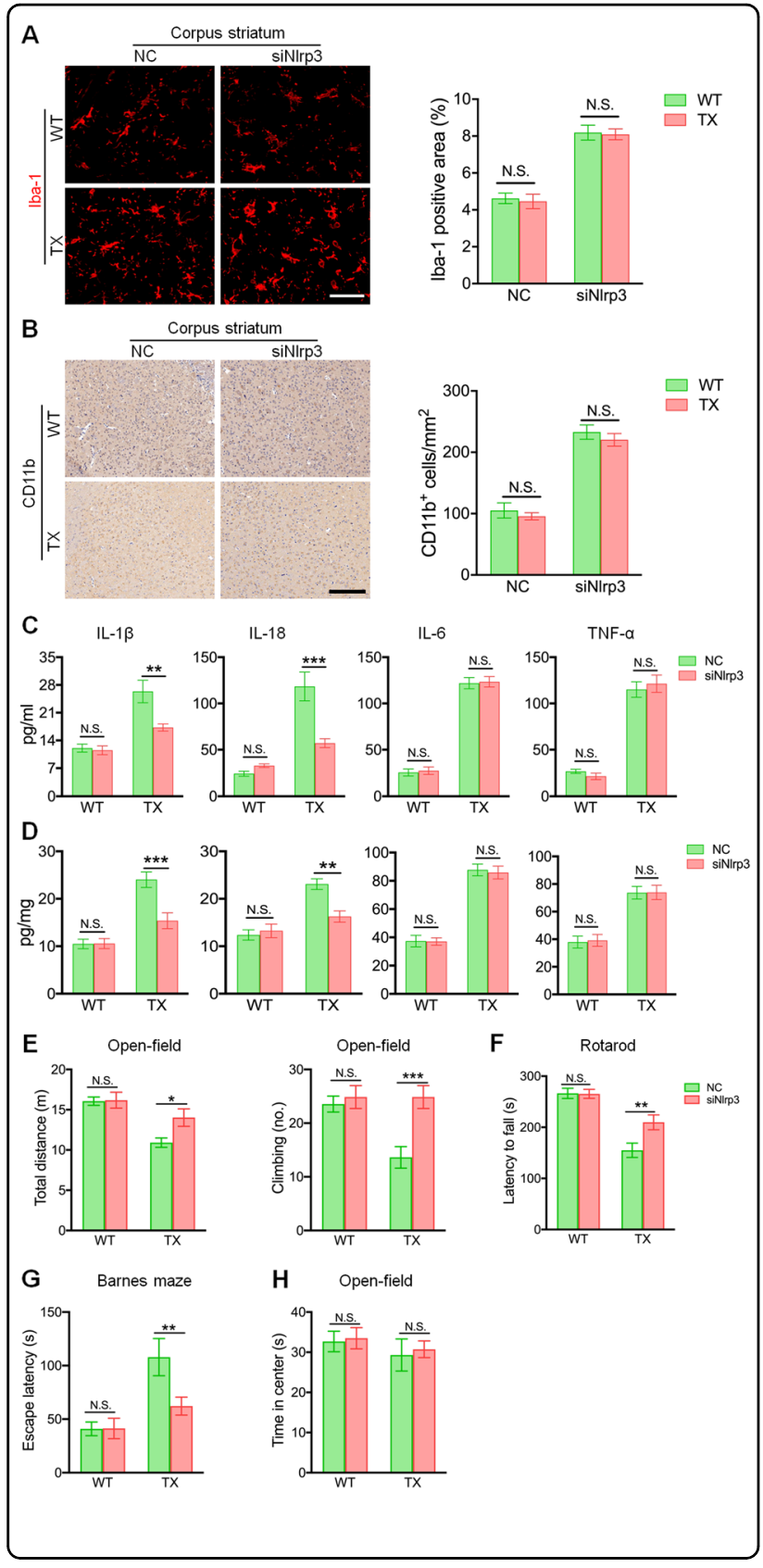

neuroinflammation reportedly plays a pivotal role in the pathogenesis of WD. In this study, we demonstrated that the NLRP3 inflammasome is actively involved in WD pathophysiology and progression. In a murine model of WD, NLRP3 silencing or pharmacologic inhibition of NLRP3 inflammasome activation attenuated the pathological progression of copper-induced injury and prevented neurodegeneration.

Acute inflammatory cytokines, such as TNF- $\alpha$, and chronic inflammatory signals, such as inflammasome activation, have been implicated in neurological inflammatory conditions. Expectedly, major NLRP3 inflammasome
Fig. 4 Nlrp3 silencing inhibits interleukin (IL)-1 $\beta$ and IL-18 production, and reverses behavioral deficits in Wilson's disease (WD) animal model. a Immunofluorescence staining and quantification of Iba-1-positive cells in the corpus striatum of the negative control siRNA (NC)- or the siNIrp3-injected toxic milk (TX) mice and wild type (WT) mice. Scale bars, $50 \mu \mathrm{m}$. $\mathbf{b}$ Immunohistochemical staining and quantification of CD11b-positive cells in the corpus striatum of the NC- or the siNIrp3-injected TX mice and WT mice. Scale bars, $200 \mu \mathrm{m}$. c Serum IL-13, IL-18, IL-6, and tumor necrosis factor (TNF-a) levels in the NC- or the siNlrp3-injected TX mice and WT mice $(\mathrm{pg} / \mathrm{ml})(n=6)$. $\mathbf{d} \mathrm{IL}-1 \beta, \mathrm{IL}-18, \mathrm{IL}-6$, and TNF-a levels in the brain of the NC- or the siNlrp3-injected TX mice and WT mice $(\mathrm{pg} / \mathrm{mg})(n=6)$. e TX mice or WT mice were treated with lentivirusenveloped siNlrp3 or control virus; activities including total distance and climbing behaviors of open-field were measured $(n=15)$. $\mathbf{f}$ TX mice or WT mice were treated with lentivirus-enveloped siNlrp3 or control virus, and after 3 months, motor functions were tested using a rotarod test apparatus $(n=15)$. $\mathbf{g}$ Escape latency was measured using Barnes maze test $(n=15)$. $\mathbf{h}$ TX mice or WT mice were treated with lentivirus-enveloped siNlrp3 or control virus, and time spent in center of open-field was measured. $(n=15)$. Data are presented as mean \pm SEM; two-way ANOVA, ${ }^{*} P<0.05,{ }^{* *} P<0.01,{ }^{* * *} P<0.001$ versus the corresponding NC group.

markers IL-1 $\beta$ and IL-18 were elevated in the sera of WD patients, suggesting an essential role for inflammasome dysregulation in the development of neuroinflammation in WD. Various types of inflammasomes, such as NLRC4, NLRP3, NLRP2, NLRP1, and AIM2, were activated in different regions of the brain in TX mice. Among these, activation of the NLRP3 inflammasome resulted in the most substantial changes. In addition, activation of the canonical NLRP3 inflammasome is more sensitive to accumulated copper in the brain, as compared to other inflammasomes. Further, a copper chelator inhibited the NLRP3 and not the AIM2, NLRC4, and NLRP1 inflammasomes or NF-kB-dependent priming ${ }^{25}$. Therefore, we speculate that $\mathrm{Cu}^{2+}$ specifically triggers the intracellular NLRP3 sensor to activate the inflammasome; however, the detailed mechanism remains to be further investigated.

It is widely accepted that successful NLRP3 inflammasome activation requires co-operation between NF-kBactivating stimuli, such as toll-like receptor ligands, to transcriptionally enhance the expression of pro-IL- $1 \beta^{29,30}$. Moreover, the activating signal is provided by various endogenous stimuli, such as PAMP or $\mathrm{Cu}^{2+}$-conjugated misfolded proteins, which activate NLRP3 ${ }^{31-33}$. Unlike several other NLRP3 inhibitors, MCC950 inhibits NLRP3induced ASC oligomerization and prevents caspase-1 cleavage into its active form, with specific selectivity ${ }^{34,35}$. Notably, the potential therapeutic value of MCC950 has been validated in vivo in numerous disease models ${ }^{36-38}$. MCC950 was found to effectively block NLRP3 inflammasome activation and protect against cognitive decline, motor deficits, and neurodegeneration in TX mice. Interestingly, MCC950 did not inhibit the expression of 

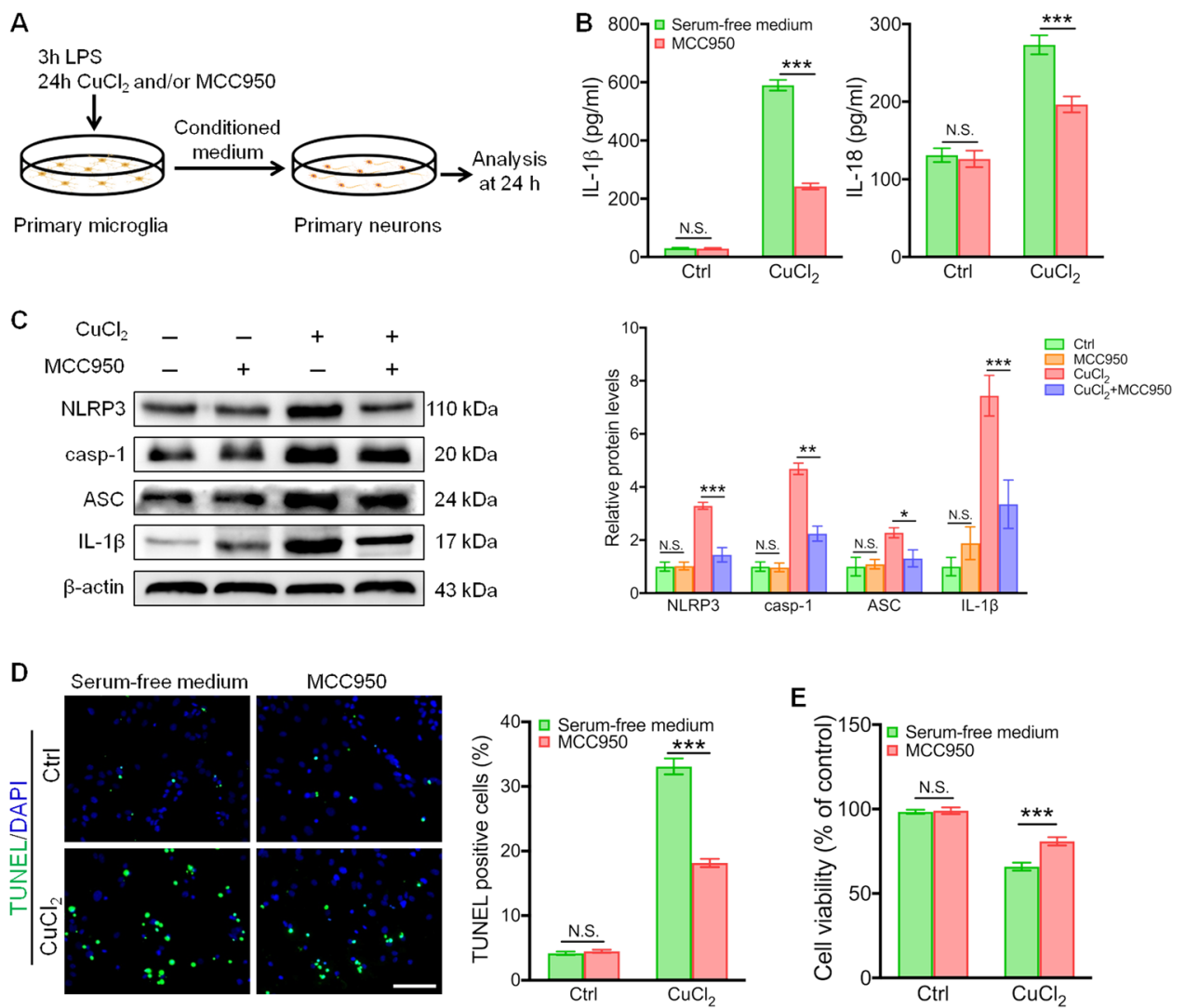

Fig. 5 MCC950 inhibits NLRP3 inflammasome activation in $\mathbf{C u C l}_{2}$-induced microglia. a Schematic illustration of the experimental set-up. $\mathbf{b}$ Primary microglia were treated with or without $\mathrm{CuCl}_{2}(10 \mu \mathrm{M})$ and $\mathrm{MCC} 950(100 \mathrm{nM})$ for $24 \mathrm{~h}$, and levels of interleukin (IL-1 $\left.\mathrm{B}\right)$ and IL-18 were quantified using ELISA $(n=6)$. c Primary microglia were treated with or without $\mathrm{CuCl}_{2}$ and MCC950, and the levels of NLRP3, cleaved caspase-1 (casp1), ASC, and IL-1 $\beta$ were measured using western blotting $(n=4)$. $\mathbf{d}$ Primary neurons separated from the hippocampus were treated with conditioned medium from $\mathrm{CuCl}_{2}$ - or MCC950-administrated microglia, and the apoptotic neuronal cells were quantified by TUNEL (green) immunofluorescence staining. Scale bars, $100 \mu \mathrm{m}$. e Cell viability of hippocampal neurons treated with conditioned medium from $\mathrm{CuCl}_{2}$ - or $\mathrm{MCC}$ (50-administrated microglia. Data are presented as mean \pm SEM; two-way ANOVA, ${ }^{*} P<0.05,{ }^{* *} P<0.01,{ }^{* * * *} P<0.001$ versus the corresponding control group.

classically secreted proinflammatory cytokines, such as TNF- $\alpha$ and IL-6; however, it only reduced unconventionally released caspase-1-dependent cytokines such as IL-1 $\beta$ and IL-18. The results demonstrate that MCC950 inhibits the assembly of the inflammasome, and this is indicated by its ability to prevent the expression of caspase 1 and ASC in the WD animal model.

Microglia, resident immune cells in the brain, could be activate by copper to induce the inflammatory response. Microglial activation is an important characteristic of the brain in WD and in animal models of WD, and the overexpression of Iba-1 indicates this change ${ }^{4,39}$. Reportedly, NLRP3 inflammasome activation plays a critical role in the initiation of microglia-mediated neuroinflammation and neuronal degeneration. Intriguingly, our analysis of microglia numbers inhibited by the activation of NLRP3 inflammasome in WD mice showed no significant differences. It is also possible that, in addition to NLRP3, other inflammasomes participate in copper-induced neuropathology.
Therefore, a combination of an inflammasome activation inhibitor, such as MCC950, and a microglial activation inhibitor, such as minocycline, may be more effective for treating neurodegeneration in TX mice.

\section{Conclusions}

Altogether, our study provides several lines of evidence to show that NLRP3 inflammasome activation is actively involved in the progression of WD and that its inhibition protects against $\mathrm{Cu}^{2+}$-induced neuroinflammation and prevents neuronal injury. Therefore, pharmacologically targeting NLRP3 inflammasome activation could be a potential therapeutic strategy for treating $\mathrm{Cu}^{2+}$-induced neuropathology in WD.

\section{Material and methods \\ Patients and controls}

Participants were recruited from the Institute of Neurology, Affiliated Hospital, Anhui University of Chinese 
A

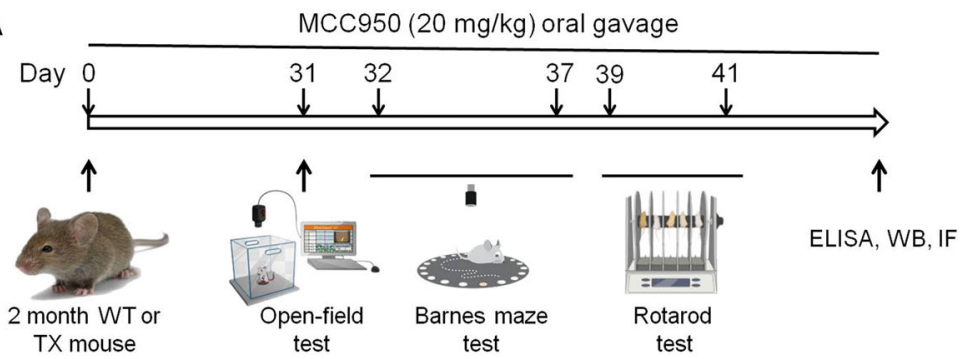

B Open-field

Open-field $\quad \mathrm{C}$

Rotarod
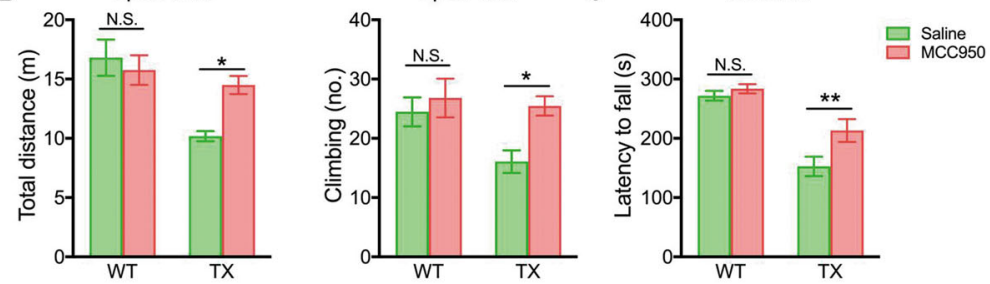

D
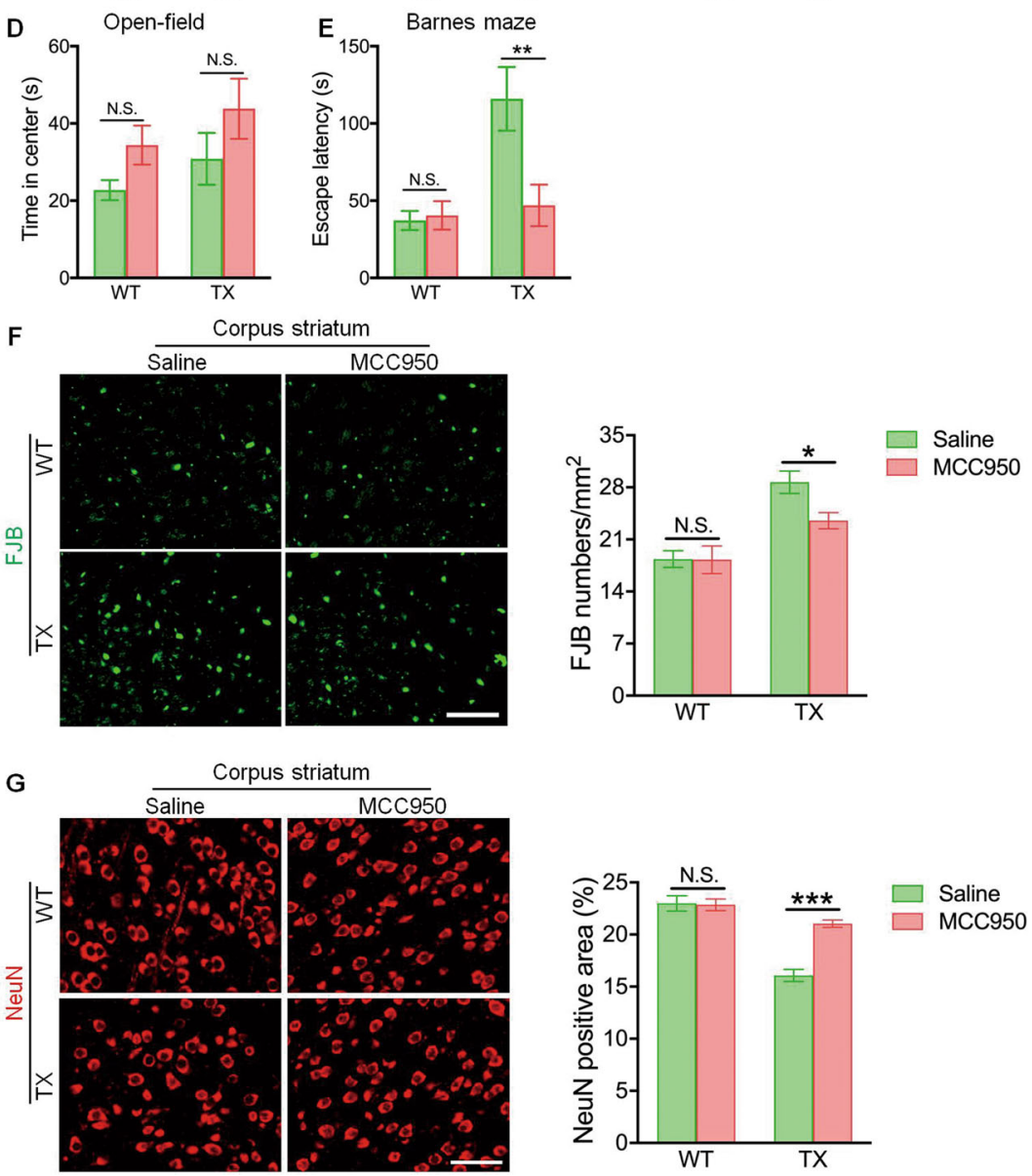

Fig. 6 MCC950 treatment protects against behavior impairment and neurodegeneration in the Wilson's disease (WD) animal model. a Schematic representation of MCC950 treatment experiments. b-e Toxic milk (TX) mice and wild type (WT) mice were treated with MCC950 or saline, (b) total distance and climbing behaviors were quantified with open-field tests $(n=15)$. c Motor functions were tested using a rotarod test apparatus $(n=15)$. $\mathbf{d}$ Time spent in center were quantified with open-field tests $(n=15)$. e Escape latency was measured using Barnes maze test $(n=15)$. $\mathbf{f}$ Fluorescence imaging of Fluoro-Jade B (FJB) staining (green) in the corpus striatum of TX mice and WT mice treated with MCC950 or saline. Scale bar, $50 \mu \mathrm{m} . \mathbf{g}$ Immunofluorescence staining and quantification of NeuN (red) in the corpus striatum of MCC950 or saline-treated TX mice and WT mice. Scale bar, $50 \mu \mathrm{m}$. Data are presented as mean \pm SEM; two-way ANOVA, ${ }^{*} P<0.05,{ }^{* * *} P<0.01,{ }^{* * *} P<0.001$ versus the corresponding saline-treated group. 

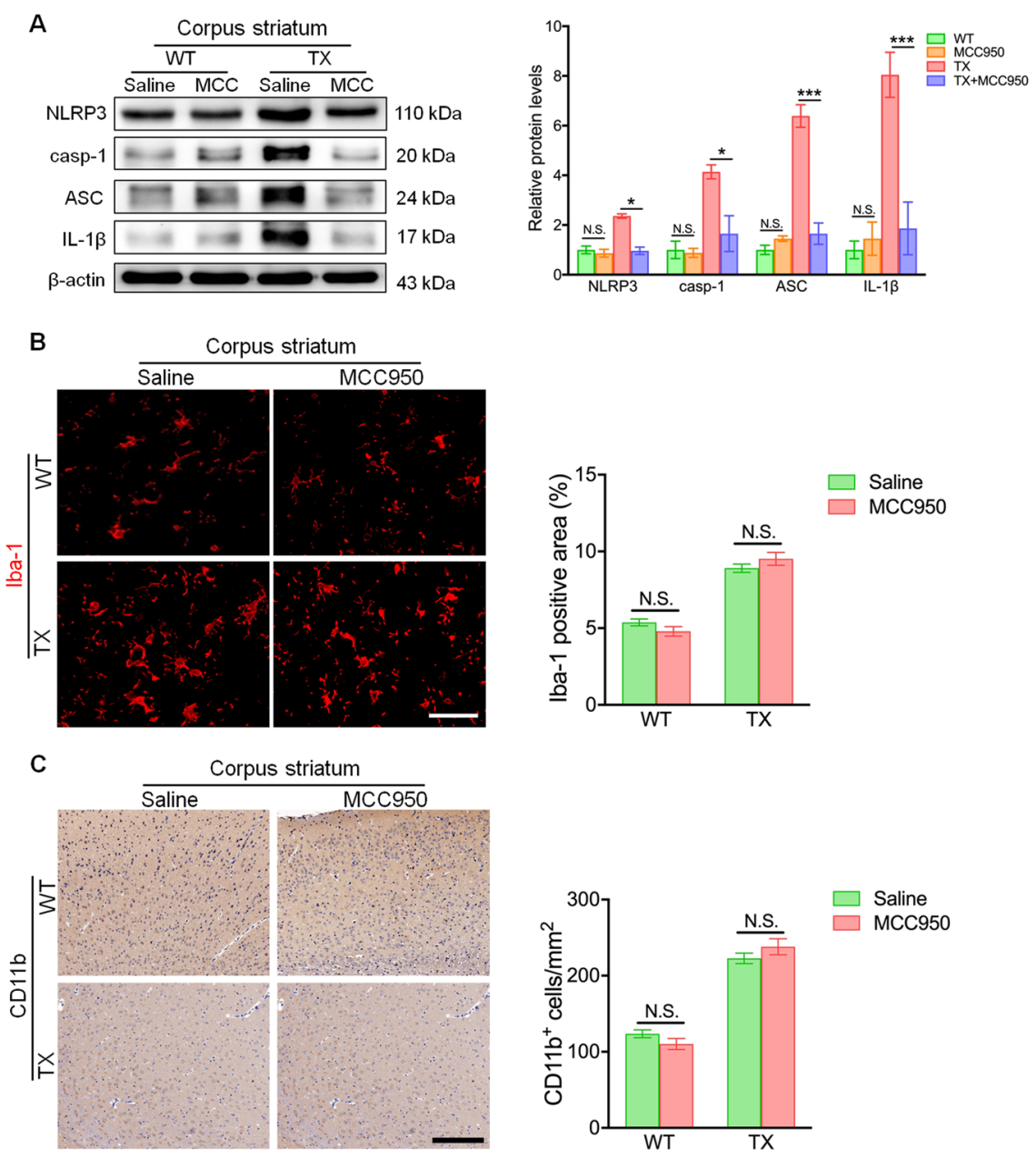

D IL-1 $\beta$

IL-18

IL-6

TNF- $\alpha$
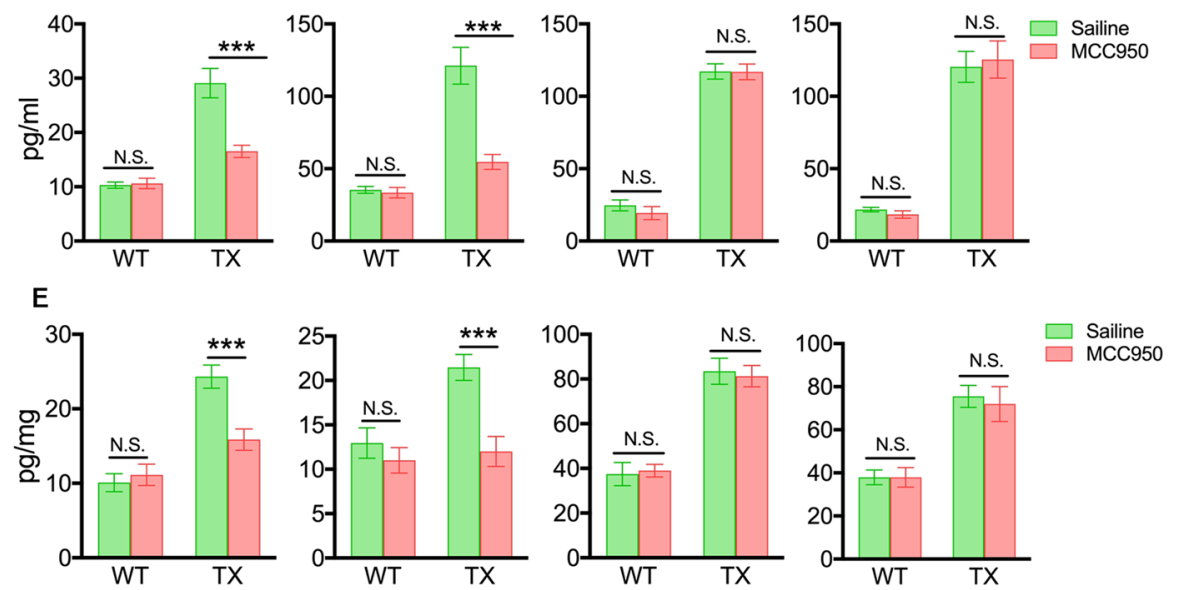

Fig. 7 MCC950 inhibits NLRP3 inflammasome activation in the Wilson's disease (WD) animal model. a Levels of NLRP3, cleaved caspase-1 (casp-1), ASC, and interleukin (IL)-1 $\beta$ in the corpus striatum of MCC950 or saline-treated toxic milk (TX) mice and wild type (WT) mice were quantified using western blotting $(n=3)$. b Immunofluorescence staining and quantification of Iba-1positive cells in the corpus striatum of MCC950 or salinetreated TX mice and WT mice. Scale bar, $50 \mu \mathrm{m}$. c Immunohistochemical staining and quantification of CD11b-positive cells in the corpus striatum of TX mice and WT mice treated with MCC950 or saline. Scale bars, $200 \mu \mathrm{m}$. d Serum IL-1 $\beta$, IL-18, IL-6, and tumor necrosis factor (TNF)- $a$ levels in TX mice and WT mice treated with MCC950 or saline $(\mathrm{pg} / \mathrm{ml})(n=6)$. e IL-1 $\beta, \mathrm{IL}-18, \mathrm{LL}-6$, and TNF-a levels in the brain of TX mice and WT mice treated with MCC950 or saline $(\mathrm{pg} / \mathrm{mg})(n=6)$. Data are presented as mean $\pm \mathrm{SEM}$; two-way ANOVA, ${ }^{*} P<0.05,{ }^{* * *} P<0.001$ versus the corresponding salinetreated group. 


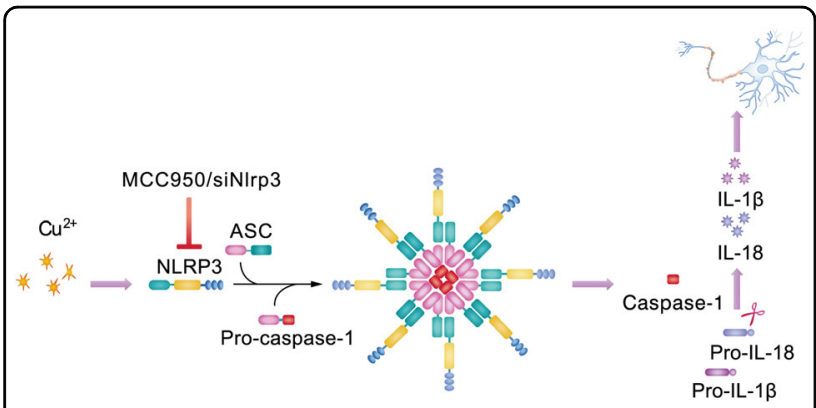

Fig. 8 Inhibiting NLRP3 inflammasome activation prevents copper-induced neuropathology in Wilson's disease (WD) animal model. NLRP3 inflammasome activation is actively involved in the progression of WD and its inhibition protects against $\mathrm{Cu}^{2+}$-induced neuroinflammation and prevents neuronal injury.

Medicine. All patients fulfilled the criteria for the clinical diagnosis of WD. Participants who had disease complications that were associated with inflammasome activation (such as depression or hypercholesterolemia) were excluded from the study. Age-matched healthy individuals (with normal serum ceruloplasmin and copper levels) were recruited as controls. Blood samples of participants were collected and centrifuged at $2500 \times g$ for $10 \mathrm{~min}$, and $500 \mu \mathrm{L}$ of the supernatant was immediately collected and stored at $-80^{\circ} \mathrm{C}$ for further analysis. All experiments were approved by and performed in accordance with the guidelines of the institutional review board of the Affiliated Hospital of the Neurology Institute of Anhui University of Chinese Medicine. Written informed consent was obtained from all participants recruited in the study.

\section{Animals}

Male and female $\mathrm{C} 3 \mathrm{HeB} / \mathrm{FeJ}$ (WT) and $\mathrm{C} 3 \mathrm{HeB} / \mathrm{FeJ}$ Atp7btx-J/J (TX) mice ${ }^{40}$ were purchased from the Jackson Laboratory (Bar Harbor, ME, USA). All mice were housed at the Hefei Institutes of Physical Science, Chinese Academy of Sciences facility under standard laboratory conditions (12-h light/dark cycle, $20-22^{\circ} \mathrm{C}, \quad 50-60 \%$ humidity), with ad libitum access to water and food throughout the study duration. All in vivo experiments were performed with 10- to 12-week-old animals; weighing $25-30 \mathrm{~g}$. Ethical approval for the animal experiments was obtained from the Hefei Institutes of Physical Science, Chinese Academy of Sciences Animal Ethics Committees. Animals were handled in accordance with the National Institutes of Health Guide for the Care and Use of Laboratory Animals.

\section{Generation of Nlrp3 knockdown mice}

For Nlrp3 knockdown, either lentivirus-enveloped Nlrp3 siRNA (siNlrp3) (Genepharma, Shanghai, China) or lentivirus-enveloped negative control siRNA $(\mathrm{NC})$ were injected through the tail vein at a dosage of $20 \mu \mathrm{L} /$ mouse $\left(10^{9} \mathrm{TU} / \mathrm{mL}\right)$. For blockade of NLRP3 inflammasome activation, $20 \mathrm{mg} / \mathrm{kg}$ of MCC950 (Selleck, Houston, TX, USA) was administered daily via oral gavage for 30 days. Control animals were given an equal volume of phosphate buffered saline (PBS).

\section{Cell culture and cell viability assay}

Primary microglia and neuron were prepared as previously described ${ }^{41,42}$. To activate the NLRP3 inflammasome, primary microglia were incubated with $200 \mathrm{ng} / \mathrm{mL}$ of ultrapure LPS (Sigma, St. Louis, USA) for $3 \mathrm{~h}$ and NLRP3 inflammasome activation was stimulated with $5 \mathrm{mM}$ adenosine 5 -triphosphate (ATP; Sigma) or $10 \mu \mathrm{M} \mathrm{CuCl}$ (Sigma) for $1 \mathrm{~h}$ (unless indicated otherwise). The microgliaconditioned media were collected, centrifuged at $900 \times g$ for $10 \mathrm{~min}$, and transferred into primary neuron culture. For the inhibition study, MCC950 $(100 \mathrm{nM})$ was added after the priming step. The survival of neurons was measured by Cell Counting Kit-8 (CCK-8; Dojindo, Kumamoto, Japan) according to the manufacturer's instruction.

\section{ELISA for inflammatory cytokines}

Mice were euthanized with an overdose of ketamine hydrochloride and transcardially perfused with $20 \mathrm{~mL}$ cold PBS as described previously prior to the removal of brain tissue ${ }^{43}$. Thereafter, brain tissues were then homogenized using an automatic grinder (Servicebio, Wuhan, China). The concentrations of inflammatory cytokines including IL-1 $\beta$ (Invitrogen, Vienna, Austria), IL-18 (Invitrogen), tumor necrosis factor (TNF- $\alpha$; Invitrogen), and IL-6 (Invitrogen) were quantified using commercial ELISA kits following the manufacturer's instructions. The absorbance was read at $450 \mathrm{~nm}$ using a microplate reader (BioTek, VT, USA).

\section{Immunohistochemical staining}

Immunohistochemical staining was performed as described previously ${ }^{44}$. Briefly, mouse brains were transcardially perfused and subsequently fixed with $4 \%$ paraformaldehyde overnight. The brain tissues were sectioned through the region of interest at a thickness of 20-30 $\mu \mathrm{m}$, and endogenous peroxidase activity was blocked with $3 \%$ $\mathrm{H}_{2} \mathrm{O}_{2}$. Thereafter, sections were incubated with anti-CD11b antibody (1:4000, Abcam, Cambridge, UK) at $4{ }^{\circ} \mathrm{C}$ overnight, rinsed three times with PBS, and incubated with the secondary antibody at $25 \pm 1{ }^{\circ} \mathrm{C}$ for $1 \mathrm{~h}$. For fluorescent staining, sections were blocked with $3 \%$ bovine serum albumin (BSA) for $30 \mathrm{~min}$, and subsequently incubated with anti-Iba-1 antibody (1:100; Abcam), and anti-NeuN antibody (1:100; Abcam) at $4{ }^{\circ} \mathrm{C}$ overnight. Thereafter, sections were rinsed thrice in PBS and incubated with fluorescent-labeled secondary antibodies at $25 \pm 1^{\circ} \mathrm{C}$ for $1 \mathrm{~h}$ in the dark. Samples were visualized using Olympus BX53 microscope (Olympus, 
Tokyo, Japan), and the images analyzed using Image J (NIH, Bethesda, MD, USA).

\section{TUNEL staining}

Apoptotic neuronal cells were quantified using Fluorescein Tunel Cell Apoptosis Detection Kit (Servicebio) following the manufacturer's instructions and as previously described ${ }^{45}$. The samples were visualized using BX53 microscope (Olympus) by an experienced pathologist blinded to the experimental design.

\section{Statistical analyses}

GraphPad Prism 8 (GraphPad, La Jolla, CA, USA) was used to perform all statistical analyses. All data were expressed as mean \pm standard error of the mean. Student's $t$ test was used for single variant analyses. The differences between means among multiple groups were compared using one-way or two-way analysis of variance (ANOVA); a $P$-value $<0.05$ was considered significant.

\section{Other methods}

Detailed description of other methods used in this study is provided in Supplementary Materials and Methods.

\section{Acknowledgements}

The authors thank Dr. Hong Zhou at Anhui Medical University for editing a draft copy of this manuscript. The work was supported by the National Natural Science Foundation of China (Grant Nos. 81603596, 81673948, 81904086, and 81774425), Natural Science Foundation of Anhui University of Chinese Medicine (Grant No. 2020sjzd03), and Natural Science Foundation of Anhui Province (Grant No. 1808085MH245).

\section{Author details}

${ }^{1}$ High Magnetic Field Laboratory, Hefei Institutes of Physical Science, Chinese Academy of Sciences, Hefei 230031, P. R. China. ${ }^{2}$ University of Science and Technology of China, Hefei 230026, P. R. China. ${ }^{3}$ The Affiliated Hospital of the Neurology Institute, Anhui University of Chinese Medicine, Hefei 230061, P. R. China. ${ }^{4}$ Anhui University of Chinese Medicine, Hefei 230012, P. R. China

\section{Author contributions}

J.J.D., Y.S.H., X.W., and N.C. designed the research theme. J.J.D. performed ELISA. J.Z., H.Y.T., and L.L.W. performed behavioral experiments and microscopy. J.J.D., S.J.W., and C.C.X. performed quantitative PCR and western blotting. M.L.G. conducted cell cultivation experiments. J.J.D. analyzed the data and plotted the figures. J.J.D. wrote the manuscript. Y.S.H. and Y.Z.H. revised the manuscript. All the authors have read and approved the final version of the manuscript.

\section{Ethics approval}

This research was approved by Hefei Institutes of Physical Science, Chinese Academy of Sciences Animal Ethics Committees (IACUC19001), and the Human Research Ethics Committee at the Affiliated Hospital of the Neurology Institute of the Anhui University of Chinese Medicine (NIMER19003).

\section{Funding}

The work was supported by the National Natural Science Foundation of China (Grant Nos. 81603596, 81673948, 81904086, and 81774425), Natural Science Foundation of Anhui University of Chinese Medicine (Grant No. 2020sjzd03), and Natural Science Foundation of Anhui Province (Grant No. 1808085MH245).

\section{Conflict of interest}

The authors declare that they have no conflict of interest.

\section{Publisher's note}

Springer Nature remains neutral with regard to jurisdictional claims in published maps and institutional affiliations.

Supplementary Information accompanies this paper at (https://doi.org/ 10.1038/s41419-021-03397-1).

Received: 13 October 2020 Revised: 28 December 2020 Accepted: 30 December 2020

Published online: 18 January 2021

\section{References}

1. Ala, A., Walker, A. P., Ashkan, K., Dooley, J. S. \& Schilsky, M. L. Wilson's disease. Lancet 369, 397-408 (2007).

2. Wu, P. et al. Inflammatory cytokines expression in Wilson's disease. Neurol. Sci. 40, 1059-1066 (2019).

3. Terwel, D. et al. Neuroinflammatory and behavioural changes in the Atp7B mutant mouse model of Wilson's disease. J. Neurochem. 118, 105-112 (2011).

4. Przybyłkowski, A. et al. Neurochemical and behavioral characteristics of toxic milk mice: an animal model of Wilson's disease. Neurochem. Res. 38, 2037-2045 (2013)

5. Mikol, J. et al. Extensive cortico-subcortical lesions in Wilson's disease: clinicopathological study of two cases. Acta Neuropathol. 110, 451-458 (2005)

6. Meenakshi-Sundaram, S. et al. Wilson's disease: a clinico-neuropathological autopsy study. J. Clin. Neurosci. 15, 409-417 (2008).

7. Latz, E., Xiao, T. S. \& Stutz, A. Activation and regulation of the inflammasomes. Nat. Rev. Immunol. 13, 397-411 (2013).

8. Lamkanfi, M. \& Dixit, V. M. Mechanisms and functions of inflammasomes. Cell 157, 1013-1022 (2014).

9. Rathinam, V. A. \& Fitzgerald, K. A. Inflammasome complexes: emerging mechanisms and effector functions. Cell 165, 792-800 (2016).

10. Place, D. E. \& Kanneganti, T. D. Recent advances in inflammasome biology Curr. Opin. Immunol. 50, 32-38 (2018).

11. Martinon, F. \& Tschopp, J. Inflammatory caspases and inflammasomes: master switches of inflammation. Cell Death Differ. 14, 10-22 (2007).

12. Franchi, L., Eigenbrod, T., Muñoz-Planillo, R. \& Nuñez, G. The inflammasome: a caspase-1-activation platform that regulates immune responses and disease pathogenesis. Nat. Immunol. 10, 241-247 (2009).

13. Mendiola, A. S. \& Cardona, A. E. The IL-1 $\beta$ phenomena in neuroinflammatory diseases. J. Neural Transm. Nienna) 125, 781-795 (2018).

14. Koprich, J. B., Reske-Nielsen, C., Mithal, P. \& Isacson, O. Neuroinflammation mediated by IL-1beta increases susceptibility of dopamine neurons to degeneration in an animal model of Parkinson's disease. J. Neuroinflammation 5, 8 (2008).

15. Sims, J. E. \& Smith, D. E. The IL-1 family: regulators of immunity. Nat. Rev. Immunol. 10, 89-102 (2010)

16. Mantovani, A., Dinarello, C. A., Molgora, M. \& Garlanda, C. Interleukin-1 and related cytokines in the regulation of inflammation and immunity. Immunity 50, 778-795 (2019).

17. Eder, C. Mechanisms of interleukin-1beta release. Immunobiology 214, 543-553 (2009).

18. Piccioli, P. \& Rubartelli, A. The secretion of IL-1 $\beta$ and options for release. Semin Immunol. 25, 425-429 (2013).

19. Lee, E. et al. MPTP-driven NLRP3 inflammasome activation in microglia plays a central role in dopaminergic neurodegeneration. Cell Death Differ. 26, 213-228 (2019).

20. Wang, Z. et al. Critical role of NLRP3-caspase-1 pathway in age-dependent isoflurane-induced microglial inflammatory response and cognitive impairment. J. Neuroinflammation 15, 109 (2018).

21. Scheiblich, $\mathrm{H}$. et al. Activation of the NLRP3 inflammasome in microglia: the role of ceramide. J. Neurochem 143, 534-550 (2017).

22. Rihel, J. Copper on the brain. Nat. Chem. Biol. 14, 638-639 (2018).

23. Hwang, J. E., de Bruyne, M., Warr, C. G. \& Burke, R. Copper overload and deficiency both adversely affect the central nervous system of Drosophila. Metallomics 6, 2223-2229 (2014).

24. de Romaña, D. L., Olivares, M., Uauy, R. \& Araya, M. Risks and benefits of copper in light of new insights of copper homeostasis. J. Trace Elem. Med. Biol. 25, 3-13 (2011). 
25. Deigendesch, N., Zychlinsky, A. \& Meissner, F. Copper regulates the canonical NLRP3 inflammasome. J. Immunol. 200, 1607-1617 (2018).

26. Gordon, R. et al. Inflammasome inhibition prevents a-synuclein pathology and dopaminergic neurodegeneration in mice. Sci. Transl. Med. 10, eaah4066 (2018).

27. Schilsky, M. L. Wilson disease: diagnosis, treatment, and follow-up. Clin. Liver Dis. 21, 755-767 (2017).

28. Roberts, E. A. Update on the diagnosis and management of Wilson disease. Curr. Gastroenterol. Rep. 20, 56 (2018).

29. Zhong, Z. et al. NF-KB restricts inflammasome activation via elimination of damaged mitochondria. Cell 164, 896-910 (2016).

30. Afonina, I. S., Zhong, Z., Karin, M. \& Beyaert, R. Limiting inflammation-the negative regulation of NF-KB and the NLRP3 inflammasome. Nat. Immunol. 18, 861-869 (2017).

31. Yang, Y., Wang, H., Kouadir, M., Song, H. \& Shi, F. Recent advances in the mechanisms of NLRP3 inflammasome activation and its inhibitors. Cell Death Dis. 10, 128 (2019).

32. Elliott, E. I. \& Sutterwala, F. S. Initiation and perpetuation of NLRP3 inflammasome activation and assembly. Immunol. Rev. 265, 35-52 (2015).

33. Jo, E. K., Kim, J. K., Shin, D. M. \& Sasakawa, C. Molecular mechanisms regulating NLRP3 inflammasome activation. Cell Mol. Immunol. 13, 148-159 (2016).

34. Coll, R. C. et al. A small-molecule inhibitor of the NLRP3 inflammasome for the treatment of inflammatory diseases. Nat. Med. 21, 248-255 (2015).

35. Coll, R. C. et al. MCC950 directly targets the NLRP3 ATP-hydrolysis motif for inflammasome inhibition. Nat. Chem. Biol. 15, 556-559 (2019).
36. Dempsey, C. et al. Inhibiting the NLRP3 inflammasome with MCC950 promotes non-phlogistic clearance of amyloid- $\beta$ and cognitive function in APP/ PS1 mice. Brain Behav. Immun. 61, 306-316 (2017).

37. Luo, Y., Lu, J., Ruan, W., Guo, X. \& Chen, S. MCC950 attenuated early brain injury by suppressing NLRP3 inflammasome after experimental SAH in rats. Brain Res. Bull. 146, 320-326 (2019).

38. Ward, R. et al. NLRP3 inflammasome inhibition with MCC950 improves diabetes-mediated cognitive impairment and vasoneuronal remodeling after ischemia. Pharm. Res. 142, 237-250 (2019).

39. Wierzba-Bobrowicz, T. et al. Morphological analysis of active microglia-rod and ramified microglia in human brains affected by some neurological diseases (SSPE, Alzheimer's disease and Wilson's disease). Folia Neuropathol. 40, 125-131 (2002).

40. Medici, V. \& Huster, D. Animal models of Wilson disease. Handb. Clin. Neurol. 142, 57-70 (2017).

41. Wu, F. et al. CXCR2 is essential for cerebral endothelial activation and leukocyte recruitment during neuroinflammationJ Neuroinflammation 12, 98 (2015).

42. Brás, J. P. et al. TNF-alpha-induced microglia activation requires miR-342: impact on NF-kB signaling and neurotoxicity. Cell Death Dis. 11, 415 (2020).

43. Shi, D. et al. FTY720 attenuates behavioral deficits in a murine model of systemic lupus erythematosus. Brain Behav. Immun. 70, 293-304 (2018).

44. Cao, K. et al. IL-33/ST2 plays a critical role in endothelial cell activation and microglia-mediated neuroinflammation modulation. J. Neuroinflammation $\mathbf{1 5}$ 136 (2018).

45. Rong, Y. et al. Neural stem cell-derived small extracellular vesicles attenuate apoptosis and neuroinflammation after traumatic spinal cord injury by activating autophagy. Cell Death Dis. 10, 340 (2019). 